Original Article

\title{
2.4 GHZ HETERODYNE RECEIVER FOR HEALTHCARE APPLICATION
}

\author{
WEI CAI ${ }^{*}{ }^{*}$, FRANK SHI ${ }^{1}$ \\ 1Department of Electrical Engineering and Computer Science, University of California, Irvine, CA, USA \\ Email: caiw2@uci.edu
}

Received: 25 Apr 2016 Revised and Accepted: 27 May 2016

\begin{abstract}
The objective of this research was to design a basic $2.4 \mathrm{GHz}$ heterodyne receiver for healthcare on a $130 \mathrm{um}$ CMOS process. The ultimate goal for the wireless industry is to minimize the trade-offs between performance and cost, and between performance and low power consumption design. In the first part, a low noise amplifier (LNA), which is commonly used as the first stage of a receiver, is introduced and simulated. LNA performance greatly affects the overall receiver performance. The LNA was designed at the $2.4 \mathrm{GHz}$ ISM band, using the cascode with an inductive degeneration topology. The second part of this thesis presents a low power $2.4 \mathrm{GHz}$ down conversion Gilbert Cell mixer. In the third part, a high-performance LC-tank CMOS VCO was designed at $2.4 \mathrm{GHz}$. The design uses using PMOS cross-coupled topology with the varactor for wider tuning range topology. In the first part, a low noise amplifier (LNA) design reaches the NF of $2 \mathrm{~dB}$, has a power consumption of $2.2 \mathrm{~mW}$, and has a gain of $20 \mathrm{~dB}$. The second part of this proposal presents a low power $2.4 \mathrm{GHz}$ down conversion Gilbert Cell mixer. The obtained result shows a conversion gain of $14.6 \mathrm{~dB}$ and power consumption of $8.2 \mathrm{~mW}$ at a $1.3 \mathrm{~V}$ supply voltage. In the third part, a high-performance LC-tank CMOS VCO was designed at $2.4 \mathrm{GHz}$. The final simulation of the phase noise is- $128 \mathrm{dBc} / \mathrm{Hz}$, and the tuning range is $2.3 \mathrm{GHz}-2.5 \mathrm{GHz}$ while the total power consumption is $3.25 \mathrm{~mW}$. The performance of the receiver meets the specification requirements of the desired standard.
\end{abstract}

Keywords: LNA, healthcare, VCO, mixer, Heterodyne receiver

(C) 2016 The Authors. Published by Innovare Academic Sciences Pvt Ltd. This is an open access article under the CC BY license (http://creativecommons.org/licenses/by/4.0/) DOI: http://dx.doi.org/10.22159/ijpps.2016v8s2.15214.

\section{INTRODUCTION}

Currently, remote monitoring applications are extremely important mobile technologies, because they can detect and prevent the illness.
Thus, they could reduce hospital readmission rates, saving hospital resources. Remote monitoring systems help patients effectively be aware of their physical conditions, and commute more efficiently gets in touch with their physicians [1]

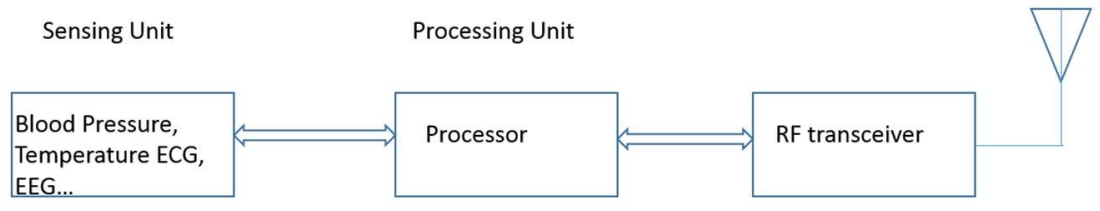

Fig. 1: Block diagram of a typical sensor node

Fig. 1 shows that the basic sensing unit can collect physiological signals (e. g.: such as electroencephalography (EEG), electrocardiography (ECG), body temperature, blood pressure, etc.), when individual sensors are attached to the human body [2]. The processing unit processes all the sensed signals, then processes all the data based on the communication protocols [3-4]. All the processed data will be transmitted through a wireless link to a portable personal base-station.

The main challenge of such remote monitoring systems is the high power consumption of portable devices. A solution to this challenge is the integration of the portable devices' digital part and the RF part onto one chip.

When the data is sent out by the transmitter, the receiver will pick up the signal and will also perform DSP processing [2]. The requirement of this transmitter and receiver is that both should have low power. For the front-end receiver, the major objectives are
1) receiving the $\mathrm{RF}$ signals and 2) recovering the biosignal classification. This paper proposes a low power receiver design. This system can be used in wireless ECG acquisition systems. In order to meet the standards, the system is designed as shown in table 1 .

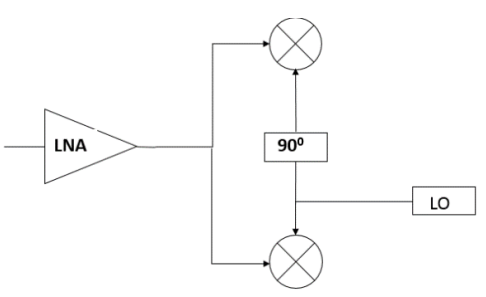

Fig. 2: Receiver topology design

Table 1: System design requirement

\begin{tabular}{llll}
\hline & NF & Gain & Power \\
\hline LNA & $3 \mathrm{~dB}$ & $15 \mathrm{~dB}$ & $<5 \mathrm{~mW}$ \\
Mixer & $16 \mathrm{~dB}$ & $5 \mathrm{~dB}$ & $<10 \mathrm{~m} \mathrm{~W}$ \\
VCO & Oscillation Freq & Phase Noise & Power \\
& $2.4 \mathrm{GHz}$ & $-100 \mathrm{dBc} / \mathrm{Hz}$ & $<5 \mathrm{~m} \mathrm{~W}$ \\
\hline
\end{tabular}


The objective of this research is to build a $2.4 \mathrm{GHz}$ Heterodyne receiver, as seen in fig. 2, which consists of an LNA, a mixer and a VCO. In this thesis, LNAs use cascode inductor degeneration topology, mixers use gilbert cells, and VCOs use LC tank topologies. All of these components use $2.4 \mathrm{GHz}$.

First, the LNA has a cascode topology and an input matching network with a series inductor to achieve a high gain of the LNA. This is shown by our simulation, which consumes only $1.7 \mathrm{~mA}$ with a $1.3 \mathrm{~V}$ power supply and achieves a gain of $20 \mathrm{~dB}$ and NF of $1.9 \mathrm{~dB}$.

Second, the mixer is a double-balanced active mixer using gilbert cell topology, which has a high conservation gain. Again our simulation results show that the mixer consumes only $6.3 \mathrm{~mA}$ with a $1.3 \mathrm{~V}$ power supply and achieves a gain of $5 \mathrm{~dB}$ and NF of $15 \mathrm{~dB}$.

Lastly, the VCO has a cross-coupled PMOS pair to achieve negative resistance, and it has a LC tank which be used to reach a resonant frequency of $2.4 \mathrm{GHz}$. Simulation results show that the VCO consumes only $2.5 \mathrm{~mA}$ with a $1.3 \mathrm{~V}$ power supply and achieves a phase noise of- $129 \mathrm{dBc} / \mathrm{Hz}, \mathrm{KVCO}$ is around $3 \mathrm{MHz} / \mathrm{V}$.

\section{MATERIALS AND METHODS}

\section{Materials}

Over the past $30 \mathrm{y}$, research on CMOS radio-frequency (RF) front-end circuits has progressed extremely quickly. The ultimate goal for the wireless industry is to minimize the trade-offs between performance and cost and between performance and low power consumption design [5].

\section{Methods}

\section{Cascode CS LNA design}

In fig. 3 , there is a cascode common-source with inductive degeneration [4]. The M1 and M2 consisted of cascode opamp and the tank circuit consisted of by $\mathrm{C}_{\mathrm{d}}$ and $\mathrm{L}_{\mathrm{d}}$. The transistor M3 was used for amplifier biasing. The purpose of inductors $\mathrm{L}_{g}, \mathrm{~L}_{\mathrm{s}}$ and capacitor $\mathrm{C}_{\mathrm{gs}}$ was to match to the 502input. The degeneration inductor $\mathrm{L}_{s}$ had better linearity at the cost of lower gain for linearity. $\mathrm{R}_{\mathrm{S}}$ was used to isolate the RF biasing signals. Other capacitors were used to block DC signals.

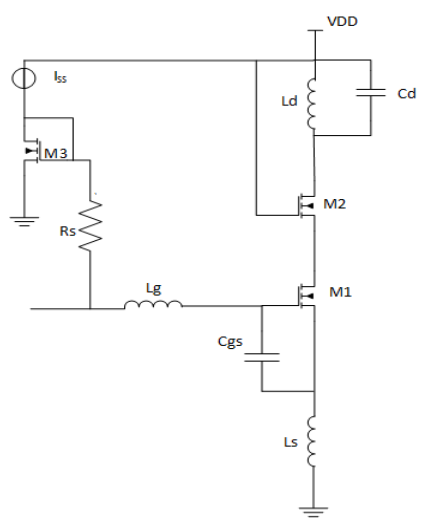

Fig. 3: 2.4GHz LNA design schematic design

The design methodology chosen is divided into the following:

Step 1: This design was based on a LNA with a $2.4 \mathrm{GHz}$ frequency. The approximated input impedance expression is

$$
Z_{i n} \approx\left(\omega L_{g}+\omega L_{s}-\frac{1}{\omega C_{g s}}\right)+\frac{g_{m 1} L_{s}}{c_{g s}}
$$

The inductive degeneration $L_{s}$ will modify the real part of the input impedance, as can be seen in (1). Thus, there is a value $L_{s}$ that corresponds to $\mathrm{R}\left\{\mathrm{Z}_{\text {in }}\right\}=50 \Omega$

$\mathrm{L}_{\mathrm{s}}$ is in series with the gate so that the imaginary part of the input impedance is zero.
Step 2: Current density is based on the minimum noise fig. (NF). Different current densities would generate different noise fig., so finding the right current density is critical. This project used the IBM $130 \mathrm{~nm}$ node, in order to calculate the noise fig. based on the process parameters.

Below is the noise power at the output of the common-source amplifier for noise due to gate resistance, drain channel noise and gate induced noise, respectively [7].

$$
\begin{gathered}
v_{n O, r_{g}}^{2} \approx 4 k T r_{g} g_{m 1}^{2} R_{L}^{2} \ldots \ldots . . . \\
v_{n o, i_{d}}^{2} \approx 4 k T \gamma g_{m 1} R_{L}^{2} \ldots \ldots . . . \\
v_{n o, i_{g}}^{2} \approx \frac{4}{5} k T \delta \omega^{2} C_{g s 1}^{2} g_{m 1} R_{L}^{2}
\end{gathered}
$$

Where $\gamma_{g}$ is the gate resistance,

$\mathrm{T}$ is the temperature; $\mathrm{k}$ is the Boltzmann constant,

$\mathrm{R}_{\mathrm{L}}$ is the amplifier load,

$\gamma$ is the excess-noise factor; its value is $2 / 3$ for long channel transistors in strong inversion,

$\omega$ is the angular frequency of the signal and

$\delta$ is a correction factor; its value is $4 / 3$ in strong inversion.

The signal power is given by

$\mathrm{P}_{\text {out }}=\mathrm{V}_{\text {out }}{ }^{2} / \mathrm{R}_{\mathrm{L}}=\mathrm{g}_{\mathrm{m}}{ }^{2} \mathrm{Vin}^{2}$

$$
\mathrm{NF}=\mathrm{P}_{\text {in }} / \mathrm{P}_{\text {out }} \ldots \ldots \ldots . . . . .(5)
$$

This equation represents the relationship between the NF and $I_{d}$. Based on Eq. (2), the noise due to gate resistance is proportional to the current, based on the Square Law Model $\left(g_{m} \propto I_{d}{ }^{1 / 2}\right)$ [8]. Since the output power is proportional to $\mathrm{g}_{\mathrm{m}}{ }^{2}$, the $\mathrm{NF}$ is not sensitive when Id changes. Like resistance gate noise, Eq. (3) and (4), are proportional to $I_{d}^{1 / 2}$. Thus, increasing the current reduces the NF. The increase in NF for higher $I_{d}$ was observed due to effects that were not considered, such as the increase of $\gamma$ with a bias current [5]. The detailed value can be referred as table 2 .

\section{LNA simulation results}

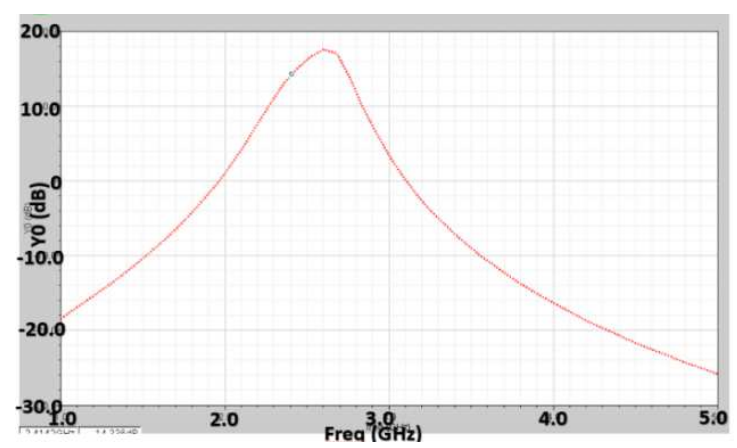

Fig. 4: Gain plot

As seen in fig. 4, the gain is $15 \mathrm{~dB}$ at $2.4 \mathrm{GHz}$.

Table 2: 2.4GHz LNA component

\begin{tabular}{ll}
\hline Parameter & Size (Unit) \\
\hline $\mathrm{M} 1, \mathrm{M} 2$ & $\mathrm{~W} / \mathrm{l}=10 \mathrm{um} / 180 \mathrm{um}$ \\
$\mathrm{M} 3$ & $\mathrm{~W} / \mathrm{l}=10 \mathrm{um} / 180 \mathrm{um}$ \\
$\mathrm{L}_{\mathrm{s}}$ & $500 \mathrm{pH}(\mathrm{Q}=20)$ \\
$\mathrm{Lg}_{\mathrm{g}}$ & $30 \mathrm{pH}(\mathrm{Q}=20)$ \\
$\mathrm{L}_{\mathrm{d}}$ & $8 \mathrm{nH}(\mathrm{Q}=20)$ \\
$\mathrm{C}_{\mathrm{gs}}$ & $120 \mathrm{fF}$ \\
$\mathrm{C}_{\mathrm{d}}$ & $440 \mathrm{fF}$ \\
$\mathrm{R}_{\mathrm{s}}$ & $10 \mathrm{~K}$ \\
\hline
\end{tabular}




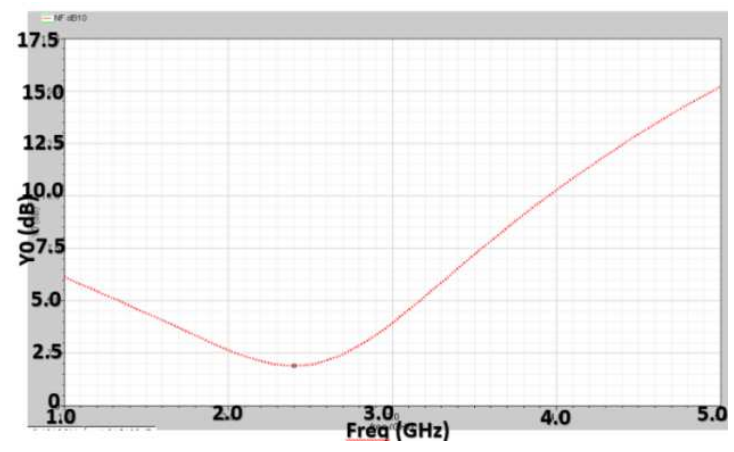

Fig. 5: NF simulation

As seen in fig. 5 , the NF in is around $1.9 \mathrm{~dB}$ at $2.4 \mathrm{GHz}$, and that the major contribution of the noise is from M1 and Ls, also, the total power of the LNA is $2.2 \mathrm{~mW}$.

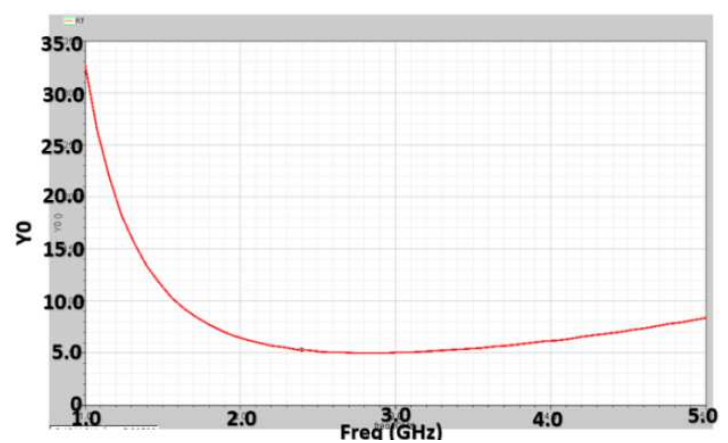

Fig. 6: Kf simulation

As seen in fig. 6, the frequency is at $2.4 \mathrm{GHz}$ the $\mathrm{S} 11$ is less than- 10 $\mathrm{dB}$. Kf is larger than 1 for all frequencies from 1 to $5 \mathrm{GHz}$, so this circuit is totally stable.

\section{Gilbert cell mixer design}

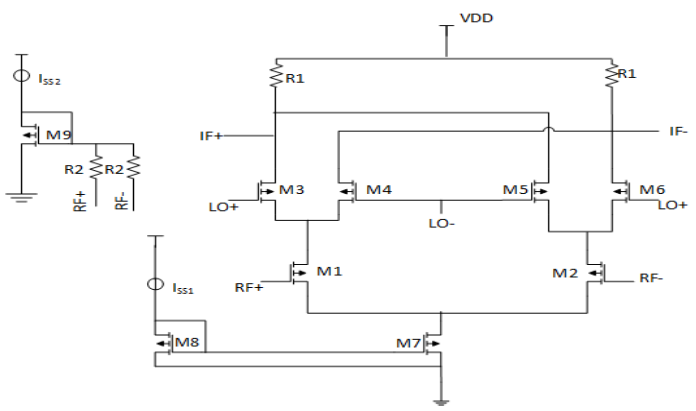

Fig. 7: 2.4 GHz gilbert mixer schematic

Gilbert mixer, as seen in fig. 7, provides good port-to-port isolation with some conversion gain [6]. The M1 and M2 are the input stages operating in the saturation region. The gain of this stage is proportional to gm, and

$$
g_{m}=k_{n}^{\prime} \frac{W}{L}\left(V_{G S}-V_{T}\right)
$$

It is obvious from the above equation that a higher overdrive voltage provides a higher gain. Increasing the width $\mathrm{W}$, while keeping the length $\mathrm{L}$ at a minimum also results in gain enhancement.
LO signals need to make the M3, M4, M5 and M6 transistors full switch, which means which transistor M3 M5 and M4 M6 are turning on alternately. In other words, LO signals must be kept at an appropriate large magnitude to ensure transistors switching accurately. Linearity is the greatly contributed decided by the trans-conductance stage.

The basic structure Gilbert cell mixer is often used in down conversion. Since transistors are operating at a saturation region, I can obtain higher gain and make the current less dependent on the changing voltage across the transistors. When I tried to bias the gain stage transistors, I needed consider whether I put enough margin of the head-room swing to make sure the transistors still working at the saturation region. The $\mathrm{LO}$ voltage level should be large enough to make the conversion gain insensitive to the LO amplitude. However, if the $\mathrm{LO}$ is too large, it will reduce the switching speed and increases the LO feed through. If two switching pair transistors conduct at the same time, noise increases. Therefore the overdrive voltage for switching pairs should be as close to zero as possible. The transistors components values can be seen in table 3 .

Table 3: 2.4GHz Mixer component

\begin{tabular}{ll}
\hline Parameter & Size (Unit) \\
\hline M1, M2 & W/l=10um/180um \\
M3, M4, M5, M6 & W/l=10um/180um \\
M7 & W/l=10um/180um \\
M8 & $W / l=10 u m / 180 u m$ \\
M9 & $W / l=10 u m / 180 u m$ \\
$C_{g s}$ & $120 \mathrm{fF}$ \\
$C_{d}$ & $440 \mathrm{fF}$ \\
$R_{s}$ & $10 \mathrm{~K}$ \\
\hline
\end{tabular}

\section{Mixer simulation results}

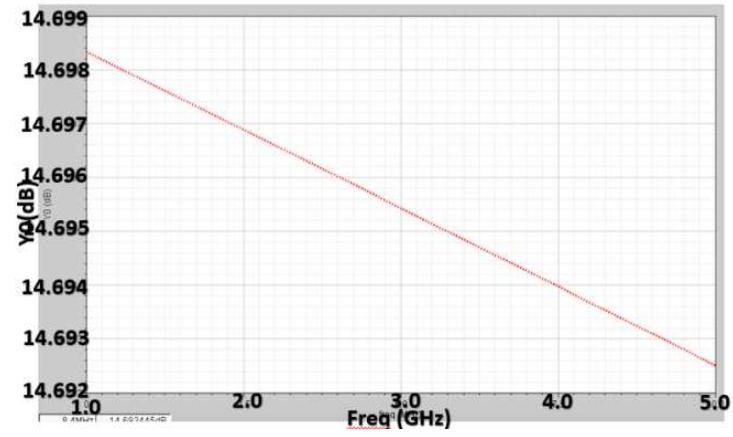

Fig. 8: NF

As seen fig. 8, based on the PSS simulation, the conversion gain VS $\mathrm{RF}$ power at $2.4 \mathrm{GHz}$ is $14.6 \mathrm{~dB}$. Also, $\mathrm{NF}$ is $15 \mathrm{~dB}$ while the power consumption is $8.2 \mathrm{~mW}$.

\section{LC VCO design}

The VCO schematic as shown in fig. 9 was chosen for several reasons. The oscillation amplitude of this structure is determined by the PMOS pair, which would also be beneficial to the low phase noise. The cross-coupled MOS pair would get a negative resistance as shown in fig. 9. The negative resistance cancels the LC element's parasitic resistance [8]. The transistor value can be seen in table 4 .

Table 4: 2.4GHz VCO component

\begin{tabular}{ll}
\hline Parameter & Size (Unit) \\
\hline M1, M2 & W/l=10um/130um \\
M3,M4,M5,M6 & W/l=1um/180um \\
M7 & W/l=4um/180um \\
C & $30 \mathrm{fF}(\mathrm{Q}=20)$ \\
L & $8 \mathrm{nH}(\mathrm{Q}=20)$ \\
C1 & W/l=10um/130um \\
\hline
\end{tabular}




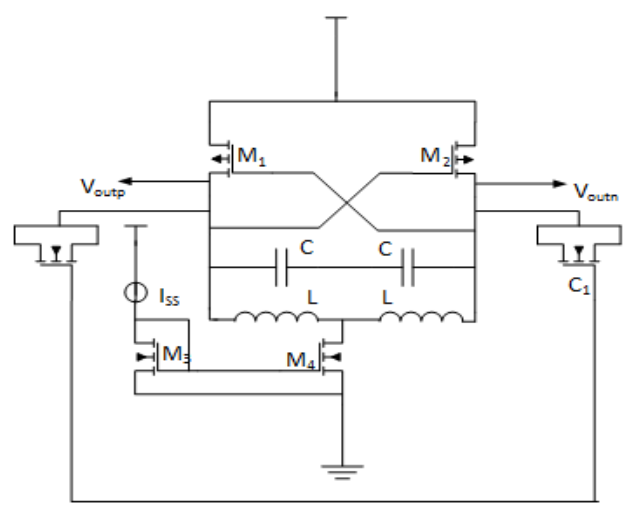

Fig. 9: 2.4 GHZ VCO schematic

\section{VCO simulation results}

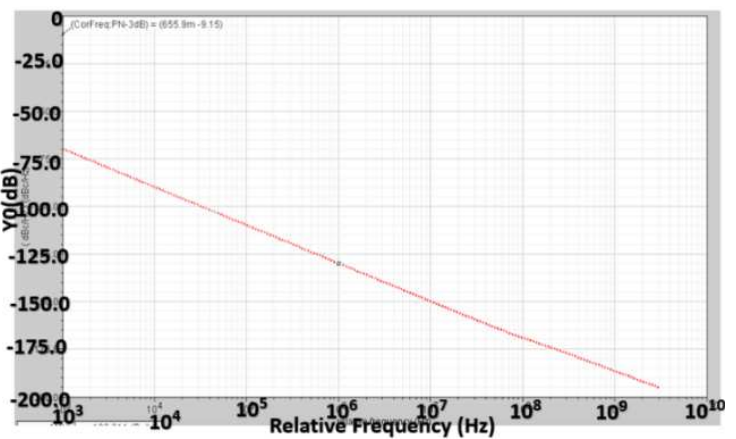

Fig. 10: VCO phase noise

The oscillation frequency is around $2.4 \mathrm{GHz}$. As we can see fig. 10 phase noise at $1 \mathrm{MHz}$ offset frequency is $129 \mathrm{dBc} / \mathrm{Hz}$, the major noise source is the current source and the two differential pair.

\section{CONCLUSION}

In hospital healthcare, the monitoring system can help doctors to monitor the patient's physiological parameters. Using proposed technology, a pregnant woman can be checked for such parameters as Blood Pressure (BP) and heart rate of the woman and heart rate and movements of fetal to control their health condition. In her proposed system, a coordinator node can be attached to a patient's body to collect all the signals from the wireless sensors and sends them to the base station. The attached sensors on a patient's body from a wireless body sensor network and they are able to sense the heart rate, blood pressure and so on. The main advantage of this system in comparison to previous systems is to reduce the energy consumption to prolong the network lifetime, speed up and extend the communication coverage to increase the freedom for enhance patient quality of life. Patients can reduce the cost of staying hospital, and doctors can monitor patients' conditions at any time. Thus, poor people can receive better healthcare, and do not have to worry about money.

This technology are extremely important because they can detect and prevent illness without a patient ever leaving their home. Thus, they could reduce hospital readmission rates, save hospital resources and save patients money.

\section{CONFLICT OF INTERESTS}

Declared none

\section{REFERENCES}

1. Stults BM. Preventive health care for the elderly. Western J Med 1984;141:832-45.

2. Hatem Trabelsi. A 3-5 GHz FSK-UWB transmitter for wireless personal healthcare applications. Int J Electronics Communications 2015;69:262-73.

3. Fan Lin. Multi-kernel learning for multivariate performance measures optimization. Neural Computing and Applications; 2016. p. 1-13.

4. Wang JB. Supervised cross-modal factor analysis for multiple modal data classification. IEEE International Conference on Systems, Man, and Cybernetics (SMC); 2015. p. 1882-8.

5. Horlin F. The generic transmission scheme for fourth generation wireless systems, WWRF Meeting; 2014. p. 1-16.

6. B Razavi. RF Microelectronics. Prentice-Hall; 1998. p. 31-67.

7. Tran Thi Thu Nga. Ultra-low-power low-noise amplifier designs for $2.4 \mathrm{GHz}$ ISM band applications. Available from: http://www3.ntu.edu.sg/home/eccboon/pub/Thesis_ThuNga. pdf $>$. [Last accessed on 10 Nov 2012].

8. B Razavi. A study of phase noise in CMOS oscillators. IEEE J Solid State Circuits 1996;31:331-43.

\section{How to cite this article}

- Wei Cai, Frank Shi. 2.4 GHZ Heterodyne receiver for healthcare application. Int J Pharm Pharm Sci 2016;8 Suppl 2:22-25. 